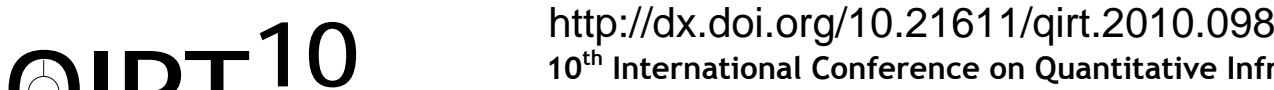 \\ $10^{\text {th }}$ International Conference on Quantitative InfraRed Thermography \\ July 27-30, 2010, Québec (Canada)
}

\section{Clinical Applications of Medical Thermography}

\author{
by Alexander Mostovoy, HD, DHMS, BCCT
}

Thermography Clinic, Inc., 143 Sheppard Avenue West, Toronto, Canada M2N 1M7, doc@drmostovoy.com

\begin{abstract}
Infrared Thermography as a medical screening tool is gaining popularity and acceptance and has been proven to be valuable in the early detection of breast disease. However, the clinical applications of infrared in the prevention of breast disease and in full body examinations have yet to be fully realized. Infrared thermography can identify thermal irregularities at a very early stage, cost effectively, and with no harm to the patient.
\end{abstract}

\section{Introduction}

At our breast thermography clinic in Toronto, we have imaged thousands of women using standardized imaging and qualitative TH reporting methods. Over the years, certain risk factors relating to breast cancer have been seen. $\mathrm{F}$ or example, our findings show that a very high percentage of women presenting with a history of breast mastectomy due to breast cancer have suggestive same-sided dental pathologies.

\section{Medical Thermography}

- An abnormal infrared image is the single-most important marker of high risk for developing breast cancer.

- A persistent abnormal thermogram carries with it a 22 times higher risk of future breast cancer.

- When thermography is added to a woman's regular breast health check-up, a $61 \%$ increase in survival rate has been realized.

- Finally, when used as part of a multimodal approach (clinical examination + mammography + thermography), 95\% of early-stage cancers will be detected.

Infrared Breast Thermography has been approved by the FDA since 1982 as an adjunctive diagnostic screening procedure for the detection of Breast Cancer. Infrared Breast Thermography measures infrared emissions from the breasts, which is then analyzed to determine a woman's risk of getting breast cancer. It is a non-invasive and safe physiological/functional imaging procedure which is underutilized in medical practice today.

The objective if this lecture will be to educate participants about available options for breast cancer screening. A comparison will be made of the risks, benefits, sensitivity and specificity between mammography and thermography. The scientific rationale and clinical approach to breast thermography will be reviewed in detail.

\section{REFERENCES}

[1] Anbar, M. "Hyperthermia of the cancerous breast: analysis of mechanism," Cancer Lett. 84, 1994

[2] Dodd, G.D., "Thermography in breast cancer diagnosis." In Proceedings of the $7^{\text {th }}$ National Cancer Conference. Los Angeles, , CA. Sept 2729 Lippincott, Philadelphia, Toronto, 1972

[3] Elliot R., Head, J., "The Important R ole of Infrared Imaging in Breast Cancer" IEEE Engineering of Medicine and Biology". May J une 2000 